\title{
Letter to the Editor on "Augmented Reality Based Navigation for Computer Assisted Hip Resurfacing: A Proof of Concept Study"
}

\author{
Ferdinando Rodriguez y Baena and He Liu (D \\ Mechatronics in Medicine Laboratory, Imperial College London, London, UK
}

(Received 23 May 2019; accepted 29 May 2019; published online 5 June 2019)

\section{Dear Editor in Chief,}

We thank Dr Cutolo for his valuable comments on our paper entitled "Augmented Reality Based Navigation for Computer Assisted Hip Resurfacing: A Proof of Concept Study". We read his letter with great interest and could not agree more with the contents. Indeed, we believe the detailed account of the current value proposition in augmented reality (AR), limits of existing technologies, and potential of current research endeavours in this space, would make for a perfect complement to the study we published in ABME, helping readers to further qualify what the impact and limitations of our work to date are.
We would also like to take this opportunity to encourage Dr Cutolo to publish an extended version of their letter as a review paper, as we have been working in this space for a few years and a comprehensive and insightful review of the AR technology space is sorely missing.

Again, many thanks for Dr Cutolo's excellent feedback. We appreciate the opportunity to have this in-depth discussion on the limitations and future development of AR technology.

Yours sincerely, Ferdinando Rodriguez y Baena and He Liu
Address correspondence to Ferdinando Rodriguez y Baena, Mechatronics in Medicine Laboratory, Imperial College London, London, UK and He Liu, Mechatronics in Medicine Laboratory, Imperial College London, London, UK. Electronic mails: f.rodriguez@imperial.ac.uk, he.liu15@imperial.ac.uk 